\title{
Une nouvelle lutte des « clashes »? Fragmentation des discours de campagne et mutations des clivages (France, 2016-2017)
}

A new "clash" struggle? Electoral soundbites and the transformation of political cleavages (France 2016-2017)

¿Una nueva «lucha de clases»? Fragmentación de los discursos políticos de campaña electoral y transformación de las oposiciones políticas durante la campaña presidencial francesa (2016-2017)

\section{Sarah Al-Matary et Chloé Gaboriaux}

\section{(2) OpenEdition}

\section{Journals}

Édition électronique

URL : https://journals.openedition.org/mots/23380

DOI : $10.4000 /$ mots. 23380

ISSN : 1960-6001

Éditeur

ENS Éditions

\section{Édition imprimée}

Date de publication : 5 juillet 2018

Pagination : 71-90

ISSN : 0243-6450

\section{Référence électronique}

Sarah Al-Matary et Chloé Gaboriaux, « Une nouvelle lutte des « clashes » ? Fragmentation des discours de campagne et mutations des clivages (France, 2016-2017) », Mots. Les langages du politique [En ligne], 117 | 2018, mis en ligne le 05 juillet 2020, consulté le 22 avril 2022. URL : http:// journals.openedition.org/mots/23380; DOI : https://doi.org/10.4000/mots.23380 


\section{Une nouvelle lutte des « clashes »? Fragmentation des discours de campagne et mutations des clivages (France, 2016-2017)}

Les travaux consacrés à la spectacularisation de la scène publique (Verón, 1995; Charaudeau, 2005), à laquelle les petites phrases sont bien souvent rattachées (Krieg-Planque, Ollivier-Yaniv, 2011), suggèrent que les techniques de communication vident le discours politique de sa substance, en convertissant le potentiel polémique des anciens clivages en «clashes » dont le retentissement dans la sphère médiatique est aussi fulgurant que fugace. C'est à cette dimension polémique de la petite phrase que le présent article est consacré. Plus précisément, il s’interroge sur la conflictualité mise en scène par la fragmentation des discours politiques dans les médias : relève-t-elle de joutes verbales qui n'aboutissent qu'à brouiller les clivages politiques, dont la dernière campagne électorale a paru confirmer l'affaiblissement? Les exprime-telle autrement, fournissant par là aux électeurs de nouveaux points de repère? En somme, le jeu des petites phrases rend-il compte des lignes de partage qui structurent l'échiquier politique français; et si oui, comment?

À partir d'une sélection d'énoncés attestés, prononcés en France pendant la campagne présidentielle de 2016-2017, et caractérisés à la fois par leur circulation et leur dramatisation médiatique, nous établirons dans quelle mesure le critère agonistique, omniprésent dans l'acception profane de la petite phrase, peut intégrer notre questionnement scientifique. Nous montrerons ensuite comment certains de ces énoncés révèlent, de façon ramassée et souvent humoristique, les mutations bien réelles des clivages politiques. Il en découle une interrogation propre au fonctionnement de ces petites phrases: comment peuvent-elles à la fois condenser les significations complexes de la division politique et donner le sentiment d'un appauvrissement du discours?

Université Lumière Lyon 2, IHRIM (UMR 5317)

almatary76@hotmail.com

Université de Lyon, Sciences Po Lyon, Triangle (UMR 5206)

chloe.gaboriaux@sciencespo-lyon.fr 


\section{De la conflictualité au carré}

\section{La dimension polémique de la petite phrase}

La définition des petites phrases n'est pas encore stabilisée. Dans le discours des acteurs, le syntagme peut renvoyer à des énoncés dont la longueurvarie et qui ne sont pas forcément des phrases au sens grammatical du terme - groupe nominal ou ensemble de phrases. Le Trésor de la langue française se contente ainsi du terme relativement vague de «propos», qu'il assortit à des éléments relatifs à son efficacité : «propos bref d'un homme politique, qui sert à frapper l'opinion ». Les travaux de recherche qui se sont intéressés à son emploi - dans la presse mais aussi chez les politiques et les communicants - montrent néanmoins que la petite phrase, si elle «frapp[e] l'opinion », n'est pas forcément intentionnelle : des énoncés considérés comme significatifs par les journalistes peuvent ainsi être extraits du discours des acteurs politiques et qualifiés de «petites phrases » sans que ces derniers l'aient toujours prévu et voulu (voir par exemple Maingueneau, 2011), et sans que l'extraction corresponde forcément à l'acception savante de la petite phrase. À cet égard, la définition qu'en propose Alice Krieg-Planque semble plus précise que celle du Trésor de la langue française : "énoncé que certains acteurs sociaux rendent remarquable et qui est présenté comme destiné à la reprise et à la circulation » (KriegPlanque, 2011). Cette définition suggère qu'un acteur politique ou médiatique peut qualifier un énoncé de «petite phrase » sans qu’il en devienne une, c'està-dire sans parvenir à le « rend[re] remarquable» ni à le faire «circul[er]». Elle est ainsi suffisamment large pour englober les divers usages que les acteurs sociaux peuvent faire du syntagme, tout en proposant des pistes d'objectivation du phénomène utiles aux analystes : c'est un énoncé coproduit, qui n'existe que par sa mise en valeur et en circulation par une pluralité d'acteurs sociaux, par le biais de procédés discursifs, médiatiques et politiques qu'il est dès lors possible d'étudier sous différents aspects.

La conflictualité en est un. Cette dernière a quelque chose d'évident pour les acteurs, comme le montrent Alice Krieg-Planque (2011) ou, ici même, Annabelle Seoane. La petite phrase est couramment associée à la polémique, et il n'est pas rare qu'elle intègre un réseau de métaphores guerrières : dans la presse, elle est moins assimilée au bon mot qu'à la pique ou la punchline - le terme s'est récemment banalisé chez les journalistes, qui l'empruntent au lexique des battles, joutes verbales rappées, lieu de compétition et de reconnaissance symbolique dans la «culture de rue» (Labov, 1978 [1972]; Vettorato, 2008). Le conflit constitue également pour les chercheurs une entrée privilégiée, en ce qu'elle permet de mieux cerner le fonctionnement de ces énoncés. La violence que les acteurs sociaux décèlent dans le jeu des petites phrases doit être en 
effet rapportée à la façon dont les médias les mettent en scène : or la dimension polémique apparaît comme l'un des critères qui président à la sélection par les journalistes de tel ou tel énoncé et favorisent son succès sur les réseaux sociaux (Leroux, Riutort, 2011), a fortiori en période électorale, quand tous les projecteurs sont braqués sur l'affrontement des candidats.

Constituer un corpus d'étude qui rende compte de cette conflictualité n'est pas chose facile. Sans base de données qu'on puisse interroger à partir d'une requête qui permettrait de rassembler tous les énoncés répondant à la définition de la petite phrase que nous avons retenue, impossible de constituer le corpus exhaustif des petites phrases de la campagne électorale et d'en tirer le sous- corpus des petites phrases polémiques pour en analyser les propriétés et les usages. Dans la mesure où notre ambition n'est pas de rendre compte du rôle des petites phrases dans cette campagne - au sens où nous nous efforcerions de déterminer et de caractériser l'ensemble de leurs usages - nul besoin d'un corpus exhaustif ni même statistiquement représentatif de ce phénomène discursif. Nous cherchons en effet à saisir le mécanisme qui fait de certaines petites phrases, peu importe leur nombre, des catalyseurs du conflit politique. Nous nous sommes donc contentées de repérer un certain nombre d'énoncés significatifs à ce titre, en ce qu'ils répondent aux critères de mise en valeur et en circulation - ou «panaphorisation », pour reprendre le terme proposé par Dominique Maingueneau (2011) - et intègrent d'une façon ou d'une autre la conflictualité politique. La plupart ont d'ailleurs été qualifiés de «petites phrases» dans la presse.

\section{La petite phrase comme catalyseur du conflit}

À cet égard, les énoncés collectés ont d'abord permis de distinguer plusieurs niveaux de conflictualité.

À un premier niveau, l'énoncé aphorisé décrit le conflit, qu'il s'agisse de caractériser les positionnements des divers protagonistes ou d'évaluer l'état du rapport de forces. C'est le cas d'une petite phrase qui a circulé jusqu'à la fin de la campagne électorale : «Macron, c'est du populisme mondain* ${ }^{1}$. En octobre 2016, lors de l'entretien politique «Les 4 Vérités» de l'émission Télématin (France 2), François Baroin attaque longuement Emmanuel Macron. Les journalistes écartent les accusations de trahison (de François Hollande) et de désertion (de l'État), mais retiennent le syntagme «populisme mondain », emprunté au passage suivant :

Il y avait, à une époque, vous vous en souvenez, un socialisme à visage humain. II y a aujourd'hui un cynisme au visage souriant doublé d'un populisme mondain, ce que Manuel Valls appelle un populisme light. (F. Baroin, Télématin, 5 octobre 2016)

1. Les énoncés suivis d'un astérisque ont été analysés par les membres de la Société d'étude des langages du politique (SELP) à l'adresse http://selp.eu/Figures/ (consulté le 16/03/2018). 
Le sémantisme de «populisme» comme son cotexte en font une étiquette politique. D'un côté, l'analogie avec « socialisme à visage humain » renvoie implicitement $\mathrm{E}$. Macron à la gauche, et pour les téléspectateurs qui connaissent l'expression, à la période soviétique. De l'autre, «populisme» le rapproche de l'extrême droite, les deux apparaissant comme les branches d'une même famille politique. Le jeu des allusions et des références historiques, condensé par le caractère oxymorique du syntagme, rappelle ainsi le fonctionnement de «totalitarisme», qui, en temps de guerre froide, avait permis d'associer nazisme et stalinisme, pourtant diamétralement opposés sur l'échiquier politique. Mais les acteurs politiques et médiatiques n'ont pas été sensibles à ces nuances, et le propos, réduit à «populisme mondain » (parfois reformulé en «populiste mondain »), a été martelé à droite jusqu'à la fin de la campagne :

4 Vérités - Baroin : «Macron est un cynique au visage souriant doublé d’un populiste mondain». (Francetvinfo.fr, 5 octobre 2016)

François Baroin accuse Macron de populisme mondain. (Challenges.fr, 5 octobre 2016)

Cette nouvelle forme de populisme mondain qui a inventé l'élection présidentielle sans programme présidentiel. (François Fillon à Poitiers, 9 février 2017)

Jean-François Copé dénonce le «populisme mondain» d’Emmanuel Macron. (Europe1.fr, 8 mars 2017)

François Baroin : «Macron, c'est du populisme mondain ». (Le Parisien, $1^{\text {er }}$ avril 2017)

L'étiquette forgée par F. Baroin a fait mouche. Elle est reprise et reformulée par les médias et apparaît dès lors à ses alliés comme un élément de langage efficace, puisqu'elle leur permet d'être aussitôt cités - c'est-à-dire rendus visibles tout en ramenant la singularité autoproclamée d'E. Macron à du connu : cette catégorie de «populisme» tant appréciée par la presse ${ }^{2}$. Elle vise ainsi à redéfinir les places dans l'affrontement politique du moment, en donnant à la droite le beau rôle : face à elle, des «populistes», dont l'un, aussitôt contredit par l'adjectif «mondain », voit son positionnement vidé de toute consistance...

D’autres énoncés aphorisés renvoient également au conflit politique, non pour en décrire les positions, mais pour caractériser les rapports de forces, les estimer et les qualifier. C'est le cas notamment de deux petites phrases que les médias ont fait circuler, l'une effectivement prononcée par Jean-Luc Mélenchon sur BFM TV le 17 février, l'autre attribuée par Le Canard enchaîné à Nicolas Sarkozy dans son édition du 15 mars 2017 :

Je n'ai pas l'intention de m'accrocher à un corbillard.* (J.-L. Mélenchon à propos du PS, BFM TV, 17 février 2017)

Fillon est mort de chez mort.* (attribué à N. Sarkozy, Le Canard enchaîné, 15 mars 2017)

2. À la mi-février 2018, plus de 19000 articles recensés par Europresse comportaient dans leur titre les termes «populisme(s) » ou « populiste(s) », dont 9451 pour la seule période 2016-2017. 
Même si la reprise des deux énoncés ne se fait pas selon la même logique - dans le premier exemple, les médias focalisent l'attention sur un passage d'une intervention publique, dans le second, ils révèlent un propos prononcé en off (en tout cas présenté comme tel) -, elle permet à chaque fois d'annoncer l'élimination probable des candidats des deux grands partis traditionnels que sont le Parti socialiste (PS) et Les Républicains (LR) en laissant la responsabilité du pronostic à des acteurs politiques. Les métaphores funèbres suggèrent en outre la violence du conflit politique en assimilant l'échec électoral à la mort du parti ou du candidat. L'affrontement est ici doublement caractérisé, dans l'évaluation du rapport de forces - alors aux dépens de B. Hamon et de F. Fillon - comme dans sa qualification - il est particulièrement violent.

Ce que montre cette série d'exemples, c'est que la conflictualité se joue aussi à un deuxième niveau, pragmatique cette fois. Décrire le conflit, c'est en effet aussi y entrer pour peser sur son issue. Les petites phrases que nous avons sélectionnées ne font pas que dire le rapport de forces, elles sont aussi des armes dans la lutte électorale, en tout cas lorsqu'elles ont été conçues par les locuteurs premiers pour être reprises dans la presse et les réseaux sociaux. En lançant la petite phrase sur le «populisme mondain » d’E. Macron, F. Baroin, on l'a vu, tente d'imposer une certaine perception de l'échiquier politique. Pour reprendre les catégories proposées par Ruth Amossy, il y a là « dichotomisation », au sens où le projet de la droite est posé comme antithétique à la rhétorique populiste, et "polarisation», au sens où les militants LR sont invités à s'opposer à l'extrême droite comme au parti En Marche!, renvoyés dos à dos dans une même réprobation à l'égard du populisme (Amossy, 2014). L'oxymore, qui tend à ôter toute consistance au positionnement macronien, contribue en outre à la « disqualification» propre au geste polémique. De la même façon, annoncer métaphoriquement la mort de son adversaire constitue une agression verbale et contribue à l'enterrer, a fortiori si la petite phrase devient virale...

Que le locuteur premier l'ait voulu ou non, la coproduction des petites phrases par les journalistes porte la conflictualité à un troisième niveau : celui de la mise en scène de la polémique dans l'espace médiatique, par des tiers qui ont un rapport plus ou moins distancié au conflit (Amossy, 2014, p. 210). En mettant en circulation la petite phrase de N. Sarkozy - «Fillon est mort de chez mort », Le Canard enchaîné réduit la position de l'ancien président de la République à un trait lapidaire, avec pour effet de dramatiser et radicaliser les divergences internes à LR. Le montage joue ici beaucoup, comme l'a montré Daniel Schneidermann à propos d'une petite phrase de J.-L. Mélenchon :

«Si vous élisez un des trois autres, vous allez cracher du sang. » Dans le feu du meeting, ça passe [...] Mais isolée, en tweet, en gros titre, avec une belle photo en contreplongée, alors là, au secours, Maximilien Ilitch revient. (Libération, 18 avril 2017) 
C'est en effet bien souvent parce qu'ils accentuent les oppositions et les mettent en scène que ces énoncés «font le buzz» - terme que Frédéric Torterat ne restreint pas à « une simple diffusion médiatique», car il désigne plutôt " une accumulation d'enchérissements diffus », " une combinaison d'amplifications qui n'a quelquefois pour objet que de maintenir une certaine intensité, quand bien même elle porterait sur un non- ou un pseudo-événement » (Torterat, 2010). Les montages photo ou vidéo, popularisés par des émissions télévisées comme «Le Petit Journal » - rubrique du Grand Journal de Canal + devenue Quotidien, surTMC - , rencontrent un succès d'autant plus vif qu'ils misent sur l'humour ou la parodie (Leroux, Riutort, 2011). Témoin cette vidéo, présentée à B. Hamon en plateau (Quotidien, 16 mars 2017), où le visage de Mélenchon, filmé lors d'une matinale d'Europe 1 , est converti en mèmes 3 grimaçants. Mis en séries et collés à d'autres images ou énoncés - politiques ou non -, ces mèmes font rire. Mais quel rapport ces confrontations forgées de toutes pièces entretiennent-elles avec les clivages politiques? Dans un contexte de fragilité des partis et de mutation du paysage politique (voir notamment Fillieule et al., 2017), on peut en effet se demander si les petites phrases ne contribuent pas, en proposant des joutes verbales déconnectées des conflits politiques, à la dépolitisation du débat public.

\section{Des joutes verbales aux conflits politiques}

Il n'est pourtant pas rare que les petites phrases renvoient à la structuration politique du pays. En témoigne, de façon quasi idéaltypique, la réception du discours prononcé par E. Macron à l'occasion de la fondation de son mouvement, En Marche! (6 avril 2016). L'énoncé qui retient avant tout l'attention des journalistes vient justement ébranler la partition traditionnelle de l'échiquier politique :

C'est un mouvement qui ne sera pas à droite, qui ne sera pas à gauche. (E. Macron, Amiens, 6 avril 2016)

Il est immédiatement reformulé pour nourrir titraille et autres tweets avant d'alimenter journaux télévisés, chaînes d’information continue et réseaux sociaux :

ni à droite ni à gauche

ni de droite ni de gauche

3. L'Oxford Dictionary donne du mème internet la définition suivante : «An image, video, piece of text, etc., typically humorous in nature, that is copied and spread rapidly by Internet users, often with slight variations. » https://en.oxforddictionaries.com/definition/meme (consulté le 16/03/2018). Sur la question des mèmes langagiers, voir notamment le dossier dirigé par Antoine Gautier et Gilles Siouffi dans la revue Travaux de linguistique (2017). 
Comme le remarquent les commentateurs4, la proposition d'E. Macron n'est pas nouvelle et renvoie à un constat partagé par les centristes depuis longtemps : il existe à la gauche du parti traditionnel de la droite, LR ex-UMP, et à la droite du parti traditionnel de la gauche, le PS, des affinités idéologiques fortes, qui allient libéralisme économique et libéralisme culturel, et qui contredisent la bipolarisation gauche-droite. Mais le contexte général - défiance à l'égard de la politique et notamment des partis - et les circonstances particulières - divisions internes au PS, faible popularité de la présidence Hollande, construction d'un ethos macronien transpartisan - lui permettent de faire son chemin.

Retouchée dès les premières dépêches, la «formule »5 est suffisamment indéterminée pour brouiller les repères : en contexte, elle évoque le centrisme, mais extraite, elle peut faire écho au slogan fasciste «ni droite ni gauche» (voir Sternhell, 1983), repris ensuite par le FN (voir notamment Crépon et al., 2015), comme au souci gaulliste de se tenir au-dessus des partis, ou à une forme d'apolitisme tenu à gauche pour un comportement de droite... Elle permet aussi de susciter les commentaires, qui se multiplient et lui confèrent plus de visibilité. Disséquée, expliquée, critiquée pour sa naïveté ou son hypocrisie, elle donne lieu à des tentatives de reformulation-récupération, comme lorsque Nathalie Kosciusko-Morizet, dans un entretien à Libération, oppose au « ni de droite ni de gauche » macronien son propre « et de droite et de gauche» (17 avril 2016). On le voit, la petite phrase d'E. Macron est productrice d'autres énoncés qui jouent la distinction et la connivence - celui de NKM est d'ailleurs immédiatement interprété par le journaliste comme un appel du pied au fondateur d'En Marche!.

L'indétermination justifie ainsi l'explication, ce qui offre à l'énoncé une formidable caisse de résonance. Son auteur est invité à plusieurs reprises à exposer sa perception du paysage politique, relayée par ses soutiens : les partis traditionnels sont exsangues, il faut dépasser la bipolarisation gauche/ droite, la seule opposition qui compte est celle qui confronte les progressistes aux conservateurs. E. Macron est alors lui-même conduit à amender la petite phrase, en se distinguant du centre, associé à la droite et à des formes partisanes (l'UDI et le Modem), ou en adoptant la forme affirmative - la référence au centre, qu'E. Macron cherche manifestement à éviter, disparaît alors :

ni de droite ni de gauche ni du centre

et de droite et de gauche

4. Voir par exemple Louis Nadau, «Macron, ni de droite ni de gauche : une vieille idée », Le Figaro.fr, 8 avril 2016.

5. Selon la définition qu'en donne A. Krieg-Planque, c'est-à-dire une formulation caractérisée par son figement, sa dimension discursive, son statut de référent social et son aspect polémique (Krieg-Planque, 2009). En reprenant l'expression «ni de droite ni de gauche», les médias intègrent une formule dans une petite phrase, la première favorisant la reprise et la circulation médiatique propre à la seconde, dont elle partage certains des traits, notamment en ce qui concerne l'aspect polémique. 
Le succès durable de l'expression et de ses variantes témoigne du caractère ambivalent de la petite phrase : elle exprime un conflit - avec la droite et surtout avec la gauche dont son auteur est issu - tout en en taisant l'enjeu; elle dit quelque chose de la recomposition du système partisan français, justement parce qu'elle est détachée de tout contenu programmatique. Elle apparaît ainsi comme l'écume des mutations politiques du moment tout en contribuant puissamment à l'ascension de celui qui en a été l'un des principaux vecteurs.

\title{
L’échiquier politique français en petites phrases
}

\author{
Les partis au risque des primaires : les petites phrases \\ sont-elles vraiment un jeu?
}

On retrouve cette ambivalence dans certaines des petites phrases qui ont circulé lors des primaires. Comme le montre Éric Treille dans le présent dossier, leur organisation contraint des candidats d'un même bord à mettre en scène leurs divergences à fleurets mouchetés, pour ne pas altérer la cohésion de leur parti. Cette configuration rend le débat très délicat, car les protagonistes doivent se positionner avec netteté, sans compromettre la campagne de leur adversaire s'il remporte la primaire. La retransmission en direct d'échanges minutés dévoile des conflits de positionnement, mais aussi des différends individuels, qui s'expriment entre autres par l'ironie. On se souvient de la fausse sollicitude de Manuel Valls à l'égard de B. Hamon :

Moi, je suis le candidat de la feuille de paie. Je ne veux pas, je ne voudrais pas que mon ami Benoît Hamon se retrouve être le candidat de la feuille d'impôt.* (M. Valls, 25 janvier 2017)

L'orateur ménage la chèvre et le chou en recouvrant d'un baume la blessure qu'il vient d'ouvrir : d'un côté, il conteste le rôle social que la gauche accorde à l'impôt, en condamnant le revenu universel; de l'autre, il feint d'atténuer son propos au moyen de la prétérition, en rappelant que son adversaire du moment est avant tout un «ami ». L'antithèse fonctionne d'autant mieux qu'elle s'appuie sur un parallélisme : malgré ses positions sur la Loi travail, $M$. Valls se place en bienfaiteur du salarié. Il reprend à cet effet une périphrase forgée par Arnaud Montebourg à l'occasion de la présentation de son programme : «Je suis le candidat du travail, de la feuille de paie, de la quittance de loyer» (4 janvier 2017).

À droite, la reproduction de la petite phrase où F. Fillon invoquait de Gaulle illustre les désaccords internes :

Qui imagine [...] le général de Gaulle mis en examen ? (F. Fillon, 28 août 2016) 
Il s’agissait alors d'anéantir N. Sarkozy, concurrent sérieux à l'élection, en le rendant indigne de se réclamer du gaullisme (la probité du Général est implicitement opposée à la malhonnêteté de l'ancien président), et donc de représenter LR (que N. Sarkozy dirigeait encore quelques jours auparavant). Elle se retourne rapidement contre F. Fillon, lorsque Luc Chatel, soutien de N. Sarkozy, lance :

Qui imagine le général de Gaulle se livrer au jeu des petites phrases? (L. Chatel, 4 septembre 2016)

Le trait, qui pousse le principe d'enchâssement jusqu'à l'absurde - une petite phrase dénonçant les petites phrases -, souligne la mesquinerie du bon mot de F. Fillon. La machine s'emballe quand «l'affaire Fillon » éclate. Le patron polémique est alors repris à l'envi, y compris hors du parti :

Vous imaginez le général de Gaulle employant Tante Yvonne à l’Élysée ?* (M. Valls, 25 janvier 2017)

Vous imaginez de Gaulle dire «je suis le candidat catholique» ?* (Henri Guaino, 16 mars 2017)

Imagine-t-on le général de Gaulle s’abstenir face à Pétain ?* (Gérald Darmanin, 26 avril 2017)

Selon une logique de surenchère, les questions rhétoriques se chargent d'une violence nouvelle, comme le signale la dernière occurrence relevée, laquelle convoque le fantasme de Vichy pour stigmatiser ceux qui, chez LR, refusent de soutenir Macron contre Le Pen. L'élection passée, le détail des altercations s'efface, mais le patron de la petite phrase, décontextualisé et recontextualisé à chaque fois, reste en mémoire. Devant la violence de la salve, qu'importe, finalement, qui a tiré le premier?

\section{Décrire et prescrire l'affrontement droite/gauche}

Face à la montée en puissance de deux partis qui, de façon très différente, affirment dépasser la vieille opposition gauche/droite, et plus encore après des primaires qui ont mis en lumière les divisions internes à LR comme au PS, plusieurs énoncés rappellent la pertinence de la bipolarisation politique traditionnelle. Au «ni de droite ni de gauche» d'E. Macron est ainsi très tôt opposé le mot de Mitterrand sur les centristes :

ni de gauche ni de gauche*

En écho à la devise macronienne, il crée un effet de surprise en substituant au terme antagonique attendu le substantif gauche, dont la répétition permet d'insister sur la négation, le centre étant alors perçu comme étranger, voire contraire à la gauche, c'est-à-dire... à droite! La petite phrase s'appuie sur un 
détournement qui prétend dévoiler le véritable sens à donner au slogan centriste : «et de droite et de droite».

Quelques jours seulement après le lancement d'En Marche!, Sud Ouest note que la «formule fait florès» (10 avril 2016). Elle circule notamment chez les commentateurs qui doutent de la capacité d'E. Macron à se soustraire aux anciens découpages politiques. Jean-Louis Bourlanges souligne ainsi la vérité de la petite phrase mitterrandienne :

C'est ce qui faisait dire à Mitterrand que la vraie devise des centristes, c'était : « $\mathrm{Ni}$ de gauche ni de gauche». (Le Figaro, 9 avril 2016)

Mais elle séduit surtout les avocats d'une gauche fidèle à ses engagements sociaux, comme le montrent les exemples suivants, qui résument l'intention pragmatique de ces reprises, toujours associées à des éléments de preuve attestant l'ancrage à droite d'E. Macron. Ce peut être :

- la mention des acteurs et des idées :

Micro collé au menton, il présente son «mouvement politique qui ne sera pas à droite, qui ne sera pas à gauche». Ou plutôt «ni de gauche,... ni de gauche». Ses fondateurs, ses soutiens, ses références et ses idées, tout arrime ce mouvement à la grande tradition du conservatisme libéral depuis Smith, Ricardo et les autres. (Blog de Juan S., Marianne.net, 10 avril 2016)

- la citation d'extraits des discours d'E. Macron :

Le zap \# 8 - Macron candidat ni de gauche... ni de gauche! (France insoumise, YouTube.fr, 13 décembre 2016)

- l'évocation d'un sondage qui place E. Macron à $61 \%$ des intentions de vote chez les sympathisants de droite :

Macron ni de gauche ni de gauche : c'est bien à droite qu'Emmanuel Macron fait des émules [...] (L'Humanité, 28 décembre 2016)

- l'accent mis sur le ralliement de François Bayrou :

Pour moi, cela clarifie les choses : il y a désormais un candidat de la droite classique et un candidat du centre-droit, c'est Emmanuel Macron [...] Vous savez, Mitterrand disait «le centre c'est ni de gauche, ni de gauche». Il était bien inspiré.* (B. Hamon, 23 février 2017)

Ainsi une petite phrase peut avoir plusieurs vies : le trait d'humour apparaît doté d'une vérité qu'on ne pourrait pas mieux formuler, mais qui appelle cependant une désambiguïsation. Indissociable de ses commentaires, sa reprise inlassable vise à révéler la réalité des clivages politiques pour mieux la faire exister. À cet égard, les petites phrases rapportées ou produites par la gauche radicale semblent avoir la particularité de puiser leur efficacité dans le rappel des conflits sociaux, comme dans la vidéo postée par la France insoumise (voir supra). Le mépris de classe attaché aux énoncés détachés des discours d’E. Macron explique leur circulation dans la « gauchosphère » (Theviot, 2014), 
comme celle des expressions qui en dénoncent la morgue, au point de faire glisser E. Macron du «centre-droit » à une position «extrême », alors qu'il se prépare à affronter $M$. Le Pen au second tour :

L'un parce que c'est l'extrême finance, l'autre parce que c'est l'extrême droite.* (J.-L. Mélenchon, 28 avril 2017)

À droite, on observe un geste analogue, quoiqu'en sens contraire, qui va parfois jusqu'à réemployer le «ni de gauche ni de gauche» pour en faire l'expression, en creux, de «la droite la plus enracinée », ultralibérale sur le plan économique et chrétienne sur le plan culturel (Paul-Marie Coûteaux, Le Figaro.fr, 27 novembre 2016):

François Fillon n'est ni de gauche ni de gauche et c'est tant mieux. (P.-M. Coûteaux, Le Figaro.fr, 27 novembre 2016)

Tout se passe comme si, à gauche, c'était la «vieille politique» (Inglehart, 1977), ancrée sur des fondamentaux socioéconomiques, qui rendait compte du succès de ces petites phrases, tandis qu'à droite, le réflexe - à la fois socioéconomique et culturel - empruntait à la politique des deux axes (Tiberj, 2012).

La campagne fourmille ainsi d'énoncés dont la saillance fait réagir la sphère médiatique. À l'automne, alors que l'élection présidentielle américaine débouche sur la victoire de Donald Trump contre Hillary Clinton, un tweet de J.-L. Mélenchon sous-entendant que seule la gauche radicale a une chance de l'emporter est largement repris : "Sanders aurait gagné » (9 novembre 2016). En février, une expression de N. Kosciusko-Morizet identifie le programme d'E. Macron à du « socialisme masqué »* (15 février 2017). Et lorsque E. Macron, élu à la présidence de la République, forme son premier gouvernement, médias et réseaux sociaux font résonner un tweet de la députée PS Karine Berger, qui ne voit dans l'équipe d’É. Philippe que «la droite, toute la droite, rien que la droite ${ }^{*}$ (17 mai 2017).

\section{La déstabilisation du système partisan : quand la petite phrase renvoie dos à dos}

Signe de la violence de la campagne, divers acteurs ont été qualifiés d'extrêmes. L'adjectif, qui désigne habituellement les marges du spectre politique, en est venu à caractériser E. Macron - «le candidat de l'extrême flou »* (V. Pécresse) - qu'on aurait plutôt tendance à placer au centre. Après la victoire du leader d'En Marche !, le gouvernement justifie sa position à l' « extrême centre » - nous reprenons l'expression à l'historien Pierre Serna (Serna, 2005) en dramatisant la passation des pouvoirs entre Bernard Cazeneuve et É. Philippe, deux Normands «violemment modérés »*. La déstabilisation du système partisan est telle que des candidats qui auraient pu se reconnaître aux 
extrêmes y situent au contraire leurs adversaires, et que les centristes euxmêmes assument l'épithète!

Le brouillage des positions est d'ailleurs entretenu par la propension des candidats à renvoyer leurs concurrents dos à dos. Marion Maréchal-Le Pen associe «Macron, Mélenchon, Hamon, Fillon» sur la base de l'onomastique, concluant : «un “on” ça ose tout, c'est à ça qu'on le reconnaît! »* (4 février 2017). Sommé de clarifier sa position sur le second tour, J.-L. Mélenchon rapproche quant à lui E. Macron et M. Le Pen, «l'un parce que c'est l'extrême finance, l'autre parce que c'est l'extrême droite ॥*, avant de les distinguer : «Marine Le Pen, c'est encore pire» (28 avril 2017). Mais la figure de style a fait son œuvre : dans leur grande majorité, les journalistes n'ont retenu que le parallélisme, enté sur une création lexicale, «l'extrême finance».

De manière plus générale, les anciens clivages sont estompés au profit de nouveaux antagonismes que les orateurs veulent favorables à leur parti : au soir du second tour, $M$. Le Pen diagnostique « une recomposition politique [...] autour du clivage entre les patriotes et les mondialistes »* (7 mai 2017) pour mieux opposer le FN à tout ce qui n'est pas lui (LREM, LR, le PS et la France insoumise). L'amalgame sert une vision complotiste de la politique, que signale l'acception polémique de «mondialiste» (Honoré, 1986).

Ces petites phrases sont donc loin d'être coupées des clivages politiques : au contraire, les joutes verbales ne cessent de les invoquer, dans une logique à la fois descriptive et prescriptive, puisque les nommer c'est aussi les faire exister. D'une certaine manière, elles contribuent à cartographier le débat politique (voir tableau page suivante).

\section{L'effet du commentaire : conflits de personnes et débats de fond}

Un révélateur de la « réalité » politique?

Les acteurs politiques s'efforcent ainsi de rendre compte des nouvelles lignes de partage en des termes qui leur sont favorables, soit qu'ils contribuent à accélérer les mutations en cours, soit qu'ils visent à réaffirmer la pertinence des anciennes divisions. Dans cet affrontement, les petites phrases sont des moyens de visibilisation d'autant plus indispensables que le temps de parole accordé à chaque candidat est réduit : elles condensent des significations sur lesquelles les uns et les autres espèrent être appelés à s'expliquer. La sphère médiatique dominante se prête plus ou moins bien à ce type de stratégie, ce qui conduit d'ailleurs certains candidats à privilégier une communication centrée sur les communautés numériques qui les soutiennent. Chez les journalistes professionnels, le souci d'éviter l'instrumentalisation et de décrypter la campagne électorale (Saïtta, 2008; Le Bart et al., 2014) conduit à mettre l'accent 


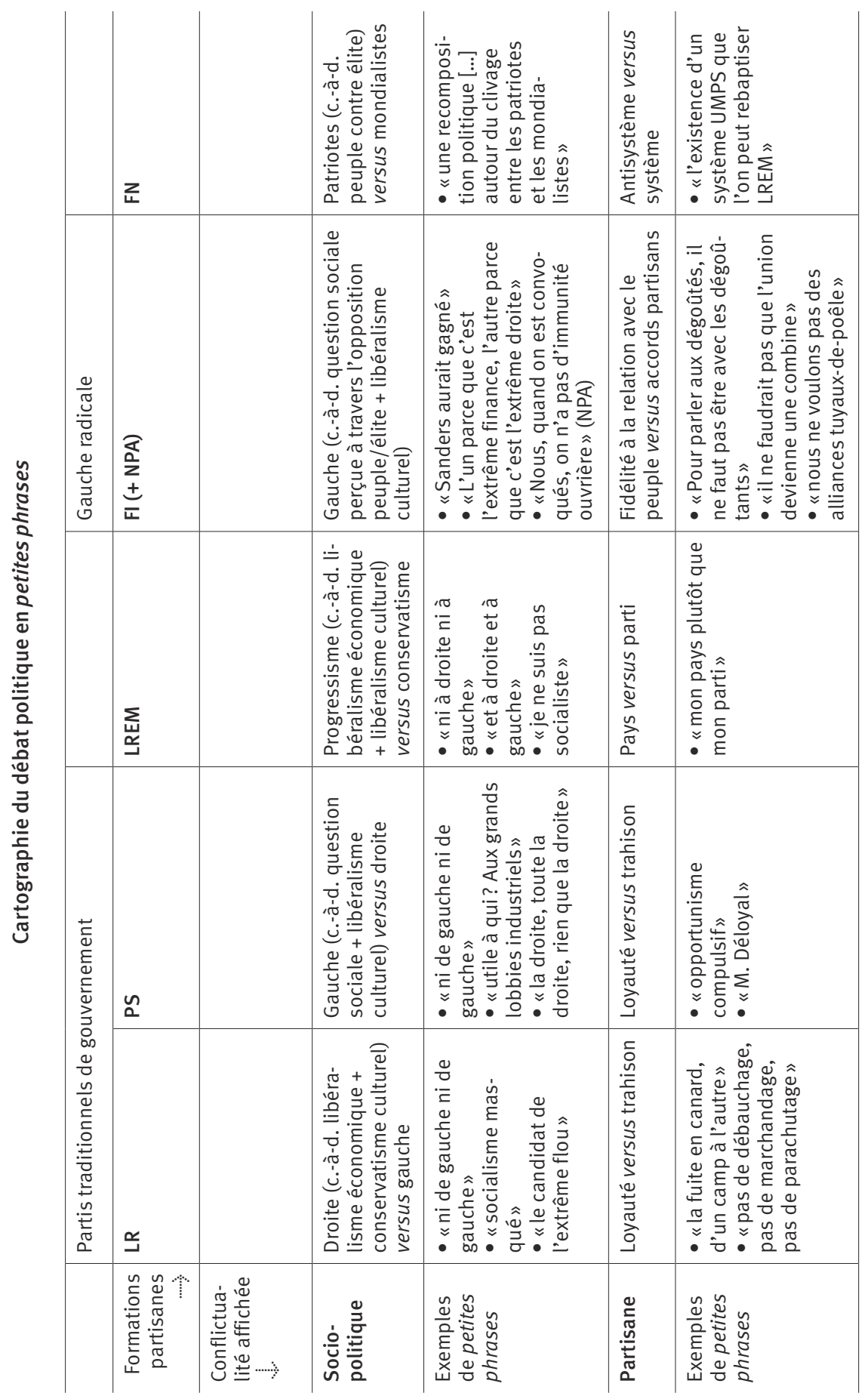


sur des énoncés qui n’ont pas été nécessairement formatés par leurs auteurs à l'intention des médias. Il s'agit alors de révéler les «coulisses» de la politique, et en particulier ce qui échappe aux déclarations de principes : la petite phrase, dans ce cas, vient révéler la «petitesse» des calculs au jour le jour.

En espérant arracher à leurs interlocuteurs un mot ou deux sur leurs intentions réelles, les journalistes suscitent des réponses vouées à être reprises et scénarisées pour mettre en lumière les arrangements politiques. On le voit avec $M$. Valls, qui opte pour l'évitement, puis la dénégation, sans parvenir à empêcher la circulation de ses réponses, commentées avec circonspection avant d'être confrontées à l'annonce de son ralliement à E. Macron fin mars :

Oui, c'est pour ça que je veux gagner! (M. Valls, sur son éventuel soutien au vainqueur, second débat de la primaire, 15 janvier 2017)

Je ne répondrai pas à cette question parce que j'en vois tous les pièges. (M. Valls sur la même question, France Info, 24 janvier 2017)

Rien dans cet article n'est vrai, en dehors du fait qu'il y a une réunion mardi. (Carlos da Silva mandaté par M. Valls, à la veille de la parution d'un article du Parisien annonçant son ralliement à E. Macron, AFP, 13 mars 2017)

La franchise ne permet d'ailleurs pas d'y échapper, comme le montrent les réactions à l'aveu de B. Hamon, confiant à Laurent Ruquier sa préférence pour J.-L. Mélenchon dans l'hypothèse d'un second tour sans le candidat PS: « Moi je vaisvous répondrefranchement: Mélenchon »,ONPP, 8 avril 2017). Marianne.net titre dès le lendemain : «Hamon s'auto-enterre».

On ne compte plus ce genre de petites phrases par lesquelles la presse prétend lever le voile sur la réalité des rapports politiques, faite de rétributions matérielles et symboliques - ce qui ne contredit pas forcément l'observation sociologique (Gaxie, 1977) - et de conflits personnels. Dans le cas qui nous occupe, elles dessinent - paradoxalement puisque la scénarisation peut paraître contraire à la logique du dévoilement - un théâtre politique fait de tractations et de trahisons : E. Macron «trahit» son parrain F. Hollande, F. Fillon «poignarde» son mentor N. Sarkozy, M. Valls est « déloyal»* à l'égard de son «ami » B. Hamon, F. Bayrou «négocie» avec E. Macron, M. Le Pen «lâche» sa nièce. Les petites phrases contribuent sans doute par là au désenchantement de la politique et même, si l'on suit Eliseo Verón, à son affaiblissement, tant elles confortent l'effacement des conflits collectifs d'ordre socioéconomique et culturel dans l'espace public en leur substituant des querelles d'ego (Verón, 1995).

\section{Tous pourris?}

Nombre de petites phrases dénoncent d'ailleurs les compromis et autres arrangements partisans. À droite, F. Fillon s'indigne ainsi des désaffections qui fragi- 
lisent son parti en fustigeant « la fuite en canard, d'un camp à l'autre, d'un hiérarque vers un autre, vers la circonscription, le portefeuille » (12 mars 2017), énoncé aussitôt repris sans que nul ne s'interroge sur la signification de l'image. Quand viennent les législatives, c'est à V. Pécresse de dénoncer les accords entre LR et LREM par une formulation en creux qui table sur l'homéotéleute pour marquer l'auditoire : «chez nous [...], pas de débauchage, pas de marchandage, pas de parachutage »* (20 avril 2017). À gauche, où l'union se fait plus pressante au fil des sondages, J.-L. Mélenchon et ses proches mettent en garde contre la «combine »* (Alexis Corbière, 14 février 2017) et les «alliances tuyaux-de-poêle » (J.-L. Mélenchon, 13 mai 2017), tandis que B. Hamon prend ses distances avec tout «opportunisme compulsif»* (10 mai 2017).

Destinés à conforter l'ethos de loyauté et d'intégrité du locuteur tout en renvoyant l'adversaire à une forme de bassesse morale, ces énoncés, dramatisés par les médias, contribuent à donner du monde politique une image dégradée, amplifiée par l'attrait des petites phrases orientées contre la «France d'en haut »* (Guillaume Tabard, 25 avril 2017). La mise en scène des vaines querelles de nos acteurs politiques suggère un conflit plus général entre les «petits» et les «grands ", les «dégoûtés » et les «dégoûtants »* - petite phrase attribuée tantôt à Pierre Mauroy, tantôt à Paul Vanden Boeynants, et remise au goût du jour par la France insoumise. Les acteurs impliqués dans le jeu des petites phrases semblent avoir été particulièrement sensibles au puissant mouvement de rejet de la politique qui travaille la société française, notamment les classes populaires (Braconnier, Dormagen, 2007) - sans être d'ailleurs forcément conscients d'y participer. L'opposition peuple/élite s'est ainsi avérée propice à la circulation de ce type d'énoncés, et pas uniquement à l'extrême droite, qui s'en est fait depuis longtemps une spécialité. Les journalistes relaient massivement la déclaration d'E. Macron affirmant « refuse[r] le système » au nom de "l'énergie du peuple français» (Bobigny, 16 novembre 2016), les références de J.-L. Mélenchon au « dégagisme »* (29 janvier 2017) sont innombrables, l'appel de F. Fillon aux électeurs contre les juges qui auraient fomenté un «assassinat politique ${ }^{*}$ (1 ${ }^{\mathrm{er}}$ mars 2017) est repris et commenté, les médias retiennent du discours de B. Hamon à Marseille sa charge contre E. Macron, en faveur de qui certains socialistes ont appelé au «vote utile» - «utile à qui ? Aux grands lobbies industriels!» (7 mars 2017).

Comme ce dernier énoncé, la petite phrase de Philippe Poutou tient en grande partie son succès de sa capacité à condenser le langage de la lutte des classes et celui de la défiance à l’égard des parlementaires :

Nous, quand on est convoqués, on n'a pas l'immunité ouvrière.* (4 avril 2017)

Retournant malicieusement le positionnement anti-système du FN contre sa présidente, empêtrée dans l'affaire des assistants parlementaires européens, P. Poutou invente une introuvable «immunité ouvrière », qui entre dans un jeu 
d'oppositions où le travailleur n'est plus le vis-à-vis du bourgeois mais de l'élu. L'énoncé trouve sa force d'attraction dans l'hybridation entre la dénonciation du mépris de classe et celle de l'élite politique.

Concurrencée par la multiplication des candidats anti-système, l'extrême droite n'a bien sûr pas renoncé à son positionnement traditionnel. À cet égard, la conjonction du centre-droit et du centre-gauche dans un même parti, En Marche!, bientôt rebaptisé La République En Marche!, corrobore à ses yeux la collusion entre les partis de gouvernement. Le hasard de la siglaison offre d'ailleurs à M. Le Pen l'occasion d'un jeu de mots qui séduit bien au-delà de la «fachosphère» (Gimenez, Voirol éd., 2017) :

La nomination sans surprise de Monsieur Édouard Philippe à la fonction de Premier ministre confirme l'existence d'un système UMPS que l'on peut rebaptiser LREM.* (15 mai 2017)

\section{Se justifier en quelques mots : grandeurs des petites phrases}

Dans la valse des petites phrases, les références aux clivages politiques côtoient ainsi le dévoilement des compromissions politiciennes, aussitôt mis en tension avec un troisième type d'énoncés, sur lequel nous conclurons cet article. Sommés de justifier leurs choix stratégiques, de préférence en quelques mots, les acteurs politiques produisent en effet des justifications, qui ordonnent les principes d'action selon une «économie de la grandeur» (Boltanski, Thévenot, 1991).

On se souvient sans doute de F. Fillon affirmant que «la France est plus grande que [s] es erreurs »* ( $1^{\mathrm{er}}$ mars 2017), ou de B. Hamon évoquant Jaurès pour se situer sur le long terme. Mais l'énoncé le plus cité et commenté en la matière est celui que la plupart des ralliés à E. Macron ont repris à leur compte, sous diverses formes : "je préfère mon pays à mon parti »*. Il semblerait que Bertrand Delanoë ait ouvert le bal. Interrogé sur les conséquences de son soutien à E. Macron pour le Parti socialiste, il conclut :

J'ai conscience, dans ma prise de position, de préférer mon pays à mon parti. (France Inter, 8 avril 2017)

Pour l'ancien maire de Paris, l'expression renvoie à un dilemme qui tient à la fois des convictions - il se sent plus proche du projet porté parE. Macron - et du réalisme politique - il le sait à même de gagner et donc de mettre en œuvre les mesures annoncées. D'où la formulation concessive, même si le choix finalement exposé est difficilement contestable : dans un climat de méfiance à l'égard des partis, et à l'heure où tous les candidats affirment pouvoir représenter l'ensemble du pays, comment pourrait-on préférer son parti à son pays? La démonstration a dû paraître particulièrement convaincante puisqu'elle est largement citée dans les médias, quoique bien souvent réduite à sa conclu- 
sion. Elle est également reprise par d'autres acteurs en délicatesse avec leur parti d'origine. Elle prend alors une tournure plus revendicative, proche de la devise ou du slogan :

Je ne sers pas mon parti mais mon pays. (H. Guaino, BFM TV, 12 mars 2017)

Je ne fais pas de la politique pour mon parti, je fais de la politique pour la France, pour mon pays. (Bruno Le Maire, RMC, 24 avril 2017)

J'ai choisi mon pays avant mon parti / plutôt que mon parti. (M. Valls, Twitter, 26 avril 2017 et CNews, 4 mai 2017)

Entre mon pays et mon parti, je ferai toujours le choix de mon pays. (Christian Estrosi, CNews, 8 mai 2017)

Mais plus que le parti, il y a le pays. (É. Philippe, TF1, 16 mai 2017)

Je préfère toujours mon pays à mon parti. (G. Darmanin, RTL, 17 mai 2017)

Je suis de droite mais je préfère mon pays à mon parti.* (Thierry Solère, Le Figaro.fr, 24 avril 2017)

Quoique commentés et plus ou moins contextualisés par les journalistes, les énoncés souffrent de la répétition : en dépit des micro-adaptations qui lui sont apportées et malgré les controverses qu'elle peut susciter (voir par exemple la critique du «slogan » par Jean-François Copé, qui le juge «écœurant», CNews, 22 mai 2017), la formule perd progressivement de son efficacité et se mue en expression stéréotypée, en cliché attestant au contraire le peu de sincérité des acteurs politiques qui la reprennent.

On l'a dit, les petites phrases que nous avons analysées sont loin de constituer un corpus exhaustif ni même représentatif : la définition labile de ces énoncés le rend introuvable. Elles sont en revanche significatives d'une certaine représentation du conflit politique, qui mêle indistinctement l'affrontement des projets et les rapports de forces sociopolitiques, les querelles de personnes et les contraintes médiatiques. La prise en compte de cette dimension essentielle au phénomène permet de dépasser l'opposition richesse/pauvreté du discours politique, qui structure encore souvent les recherches. Ces énoncés susceptibles d'être détachés et mis en circulation se distinguent en effet toujours par leur épaisseur sémantique, ce qui explique l'attrait qu'ils suscitent. C'est leur parcours médiatique qui tend à les appauvrir, aux yeux même de ceux qui contribuent à leur diffusion. Tel est peut-être - mais il faudrait élargir l'enquête pour le démontrer - le destin des petites phrases, repérées pour la densité de leurs significations, et répétées jusqu’à épuisement du sens. 


\section{Références}

Amossy Ruth, 2014, Apologie de la polémique, Paris, PUF.

BoltANSKI Luc, ThÉVENOT Laurent, 1991, De la justification : les économies de la grandeur, Paris, Gallimard.

BRACONNIER Céline, Dormagen Jean-Yves, 2007, La démocratie de l'abstention : aux origines de la démobilisation électorale en milieux populaires, Paris, Gallimard.

Charaudeau Patrick, 2005, Le discours politique: les masques du pouvoir, Paris, Vuibert.

CRÉPON Sylvain, DÉzÉ Alexandre, MAYER Nonna, 2015, Les faux-semblants du Front national: sociologie d'un parti politique, Paris, Presses de Sciences Po.

FILlieULe Olivier, HAEgel Florence, HAMIDI Camille, TIBERJ Vincent, 2017, Sociologie plurielle des comportements politiques : je vote, tu contestes, elle cherche..., Paris, Presses de Sciences Po.

GaUtier Antoine, Siouffi Gilles éd., 2016, Les mèmes langagiers : propagation, figement et déformation [numéro thématique], Travaux de linguistique, nº 73.

GAXIE Daniel, 1977, «Économie des partis et rétributions du militantisme», Revue française de science politique, $27^{\mathrm{e}}$ année, n ${ }^{0} 1$, p. 123-154.

GIMENEZ Elsa, VoIRol Olivier éd., 2017, L'Internet des droites extrêmes [numéro thématique], Réseaux, n²02-203.

Honoré Jean-Paul, 1986, "La "hiérarchie des sentiments" », Mots, n¹2, p.129-157.

In GLeHART Ronald, 1977, The Silent Revolution: Changing Values and Political Styles among Western Publics, Princeton, Princeton University Press.

KRIEG-PLANQUe Alice, 2011, "Les “petites phrases” : un objet pour l'analyse des discours politiques et médiatiques», Communication \& langages, $n^{\circ} 168$, p. $23-41$.

- 2009, La notion de «formule » en analyse du discours : cadre théorique et méthodologique, Besançon, Presses universitaires de Franche-Comté.

Krieg-Planque Alice, Ollivier-Yaniv Caroline éd., 2011, Les «petites phrases » en politique [numéro thématique], Communication \& langages, $\mathrm{n}^{\circ} 168$.

LABOV William, 1978 [1972], Le parler ordinaire : la langue dans les ghettos noirs des États-Unis, Paris, Minuit.

Le BART Christian, Leroux Pierre, Ringoot Roselyne, 2014, "Les livres de journalistes politiques. Sociologie d'un passage à l'acte», Mots. Les langages du politique, $\mathrm{n}^{0}{ }^{104}$, p. 5-17.

LeRouX Pierre, RIUTORT Philippe, 2011, "Les émissions de divertissement, de nouveaux lieux de valorisation des petites phrases », Communication \& langages, n ${ }^{0} 168$, p. $69-80$.

Maingueneau Dominique, 2011, "Sur une petite phrase “de” Nicolas Sarkozy. Aphorisation et auctorialité », Communication \& langages, n 168 , p. 43-56.

SAÏTTA Eugénie, 2008, «Les journalistes politiques et leurs sources. D’une rhétorique de l'expertise critique à une rhétorique du “cynisme" ", Mots. Les langages du politique, no 87, p. 113-128.

SERNA Pierre, 2005, La république des girouettes. 1789-1815 et au-delà. Une anomalie politique : la France de l'extrême centre, Paris, Champ Vallon.

Stern Hell Zeev, 1983, Ni droite ni gauche : l'idéologie fasciste en France, Paris, Seuil.

THEVIOT Anaïs, 2014, Mobiliser et militer sur Internet: reconfiguration des organisations partisanes et du militantisme au Parti Socialiste et à l'Union pour un Mouvement Populaire, thèse de doctorat, IEP de Bordeaux. 
TIBERJ Vincent, 2012, «La politique des deux axes. Variables sociologiques, valeurs et votes en France (1988-2007) ", Revue française de science politique, vol. LXII, nº 1 , p. 71-196.

TORTERAT Frédéric, 2010, «Quand la publicité politique se confronte au buzz journalistique : le cas des dérapages verbaux traités dans une rubrique de quotidien », Signes, Discours et Sociétés, no 5, http://www.revue-signes.info/document.php?id=1807 (consulté le 16 mars 2018).

VERón Eliseo, 1995, «Médiatisation du politique. Stratégies, acteurs et construction des collectifs », Hermès, nº 17-18, p. 201-214.

Vettorato Cyril, 2008, Un monde où l'on clashe. La joute d'insultes dans la culture de rue, Paris, Éditions des archives contemporaines.

\section{Résumé / Abstract / Compendio}

\section{Une nouvelle lutte des «clashes» ? Fragmentation des discours de cam- pagne et mutations des clivages (France, 2016-2017)}

Cet article s'interroge sur la conflictualité mise en scène par la fragmentation des discours politiques dans les médias : relève-t-elle de joutes verbales étrangères aux clivages politiques ou les exprime-t-elle autrement, fournissant par là aux électeurs de nouveaux points de repère? À partir d'un corpus ouvert, constitué d'énoncés prononcés en France pendant la campagne présidentielle de 2016-2017, et caractérisés à la fois par leur circulation et leur dramatisation médiatique, l'article démontre que les petites phrases révèlent, de façon ramassée et souvent humoristique, les mutations bien réelles du paysage politique français, mais qu'elles le font dans une ambivalence constitutive, en condensant les significations complexes de la division politique tout en donnant le sentiment d'un appauvrissement du discours politique.

Mots-clés : petite phrase, conflictualité, clivages, spectacularisation, médias

\section{A new "clash" struggle? Electoral soundbites and the transformation of poli- tical cleavages (France 2016-2017)}

This article examines how soundbites dramatize political conflicts in the media: are they independent of real political cleavages or do they refer to them otherwise, thus providing voters with new points of reference? From an open corpus of statements uttered in France during the 2016-2017 presidential campaign, characterized by their circulation and dramatization in the media, this article shows that electoral soundbites reveal real changes in the French political landscape in a constitutive ambivalence, both condensing the complex meanings of political division and contributing to the impoverishment of political discourse.

Keywords: soundbite, conflictuality, political cleavages, spectacularization, media 


\section{¿Una nueva «lucha de clases»? Fragmentación de los discursos políticos de campaña electoral y transformación de las oposiciones políticas durante la campaña presidencial francesa (2016-2017)}

Este artículo cuestiona la conflictividad escenificada por la fragmentación de los discursos políticos en los medios de comunicación: ¿se tratará de peleas verbales ajenas a las oposiciones políticas, o más bien de una otra forma de expresarlas, proporcionando a los electores nuevos puntos de referencia? Partiendo de un corpus abierto, constituido por declaraciones pronunciadas en Francia durante la campaña presidencial del 2016-2017, y caracterizadas a la vez por su circulación y su dramatización, este trabajo demuestra que las frases cortas (también llamadas frases con gancho) revelan, de manera fornida y muchas veces humorística, verdaderas mutaciones del paisaje político francés, y que lo hacen con una ambivalencia constitutiva, condensando los significados complejos de la división política y dando así la sensación de un empobrecimiento del discurso político.

Palabras claves: frase corta, conflictividad, divisiones políticas, espectacularización, medios de comunicación 